\title{
Pacific
}

Journal of

Mathematics

\section{A DISTANCE FORMULA FOR ALGEBRAS ON THE DISK}

\section{CHRISTOPHER J. BISHOP}




\title{
A DISTANCE FORMULA FOR ALGEBRAS ON THE DISK
}

\author{
Christopher J. Bishop
}

\begin{abstract}
Suppose $H^{\infty}(\mathbb{D})[\mathcal{F}]$ is the closed algebra on the disk generated by $H^{\infty}(\mathbb{D})$ and a countable collection $\mathcal{F}$ of bounded harmonic functions. Given $g \in L^{\infty}(\mathbb{D})$ we give a method for calculating the distance from $g$ to $H^{\infty}(\mathbb{D})[\mathcal{F}]$ (in the $L^{\infty}$ norm). As applications we compute the Bourgain closures of such algebras and give a new proof of a result of Axler and Shields.
\end{abstract}

\section{Introduction.}

Let $H^{\infty}(\mathbb{D})$ denote the algebra of bounded holomorphic functions on the unit disk, $\mathbb{D}$. If $\mathcal{F}$ is a collection (finite or countable) of bounded harmonic functions on the disk let $H^{\infty}(\mathbb{D})[\mathcal{F}]$ denote the algebra generated by $H^{\infty}(\mathbb{D}) \cup$ $\mathcal{F}$ (i.e., the smallest algebra containing $H^{\infty}(\mathbb{D}) \cup \mathcal{F}$ which is closed with respect to the supremum norm on $\mathbb{D}$ ). The purpose of this paper is to give a method for estimating $\operatorname{dist}\left(g, H^{\infty}(\mathbb{D})[\mathcal{F}]\right.$ ) (in the $L^{\infty}$ norm) for any $g \in L^{\infty}(\mathbb{D})$.

Let $\mathcal{M}$ be the maximal ideal space of $H^{\infty}(\mathbb{D})$. This is a compactification of $\mathbb{D}$ to which every bounded harmonic function has a continuous extension (see [6] for the definition and basic facts). Suppose $f$ is a real valued, bounded harmonic function on $\mathbb{D}$ and let $f$ also denote its continuous extension to $\mathcal{M}$. For each $a \in \mathbb{R}$ let $E_{a}=\{\zeta \in \mathcal{M}: f(\zeta)=a\}$. Then Shilov's theorem (e.g., Theorem III.2.20 of [13]) in this case implies that for any $g \in C(\mathcal{M})$,

$$
\operatorname{dist}\left(g, H^{\infty}(\mathbb{D})[f]\right)=\sup _{a \in \mathbb{R}} \operatorname{dist}\left(\left.g\right|_{E_{a}},\left.H^{\infty}(\mathbb{D})\right|_{E_{a}}\right) .
$$

In particular, $g \in H^{\infty}(\mathbb{D})[f]$ iff for every $a \in \mathbb{R},\left.\left.g\right|_{E_{a}} \in H^{\infty}(\mathbb{D})\right|_{E_{a}}$.

We will extend this in several ways. Roughly speaking,

(1) We will formulate the distance formula in a way that makes sense for all $g \in L^{\infty}(\mathbb{D})$, not just those with a continuous extension to $\mathcal{M}$. Unfortunately, this reformulation looks much messier since we can no longer refer to $\left.g\right|_{E}$ where $E \subset \mathcal{M}$, but must state everything in terms of open sets in the disk.

(2) We will consider complex valued harmonic functions. This introduces further complications because the distance formula now involves level sets of auxiliary functions instead of the original generating set. 
(3) We will allow finite and countable infinite collections of functions. Instead of level sets of individual functions we will consider joint level sets, but otherwise nothing much changes.

(4) We will allow certain generating functions in $C(\mathcal{M})$ which need not be harmonic. However, the class of functions we allow is very closely related to harmonic functions.

The precise statements require some more notation and will be given in the following sections. I thank Joe Cima, Pam Gorkin, Peter Jones, Karel Stroethoff and Tom Wolff for very helpful comments and conversations: on various aspects of this paper. This paper is a shortened version of an earlier preprint entitled "Some characterizations of algebras on the disk" which also recovered several known results using the techniques in this paper. Many errors in the original version have been corrected due to the suggestions of the referees. They have greatly improved the quality of the paper and I am very grateful for their help.

\section{The distance formula for algebras with one generator.}

In this section we state Theorem 2.1 which gives the formula for $\operatorname{dist}\left(g, H^{\infty}(\mathbb{D})[f]\right)$ where $f$ is a single harmonic function on $\mathbb{D}$ and $g \in L^{\infty}(\mathbb{D})$. For many applications, this is the most interesting case. In the next section we will give the statement for algebras with more than one harmonic generator.

If $f$ is a bounded harmonic function write $f=u+i v=\operatorname{Re}(f)+i \operatorname{Im}(f)$ and set

$$
h=\frac{1}{2}\left(\left(u+v^{*}\right)-i\left(v-u^{*}\right)\right)=\frac{1}{2}\left(\bar{f}+i \bar{f}^{*}\right),
$$

(the "*" denotes harmonic conjugation, normalized so $f^{*}(0)=0$ ). Then $h$ is holomorphic and, though it need not be bounded, it is in BMOA(D) (see [8] for definition). Moreover, $h$ is constant iff $f$ is holomorphic.

Given a function $f$ on the disk, $a \in \mathbb{C}$ and $\delta>0$ let

$$
\Omega_{f}(a, \delta)=f^{-1}(D(a, \delta))=\{z \in \mathbb{D}:|f(z)-a|<\delta\} .
$$

For convenience, given an open set $\Omega \subset \mathbb{D}$ we will write

$$
\operatorname{dist}\left(g, H^{\infty}(\Omega)\right)=\inf _{G \in H^{\infty}(\Omega)}\left\|\left.g\right|_{\Omega}-G\right\|_{L^{\infty}(\Omega, d x d y)} .
$$

If $\Omega$ is empty, we interpret the distance as 0 .

Theorem 2.1. If $f$ is a bounded harmonic function on $\mathbb{D}$ and $g \in L^{\infty}(\mathbb{D})$ then

$$
\operatorname{dist}\left(g, H^{\infty}(\mathbb{D})[f]\right)=\inf _{\delta>0} \sup _{a \in \mathbb{C}} \operatorname{dist}\left(g, H^{\infty}\left(\Omega_{h}(a, \delta)\right)\right)
$$


If we assume $f$ is anti-holomorphic then $h=\bar{f}$ so $\Omega_{h}(a, \delta)=\Omega_{f}(\bar{a}, \delta)$ and hence Theorem 2.1 is valid with $\Omega_{h}$ replaced with $\Omega_{f}$. In this case we obtain a more natural looking result.

Corollary 2.2. If $\bar{f} \in H^{\infty}(\mathbb{D})$ and $g \in L^{\infty}(\mathbb{D})$ then

$$
\operatorname{dist}\left(g, H^{\infty}(\mathbb{D})[f]\right)=\inf _{\delta>0} \sup _{a \in \mathbb{C}} \operatorname{dist}\left(g, H^{\infty}\left(\Omega_{f}(a, \delta)\right)\right) .
$$

Constant functions are examples of holomorphic functions, so one trivial (but useful) special case is

Corollary 2.3. If $\bar{f} \in H^{\infty}(\mathbb{D})$ and $g \in L^{\infty}(\mathbb{D})$ then

$$
\operatorname{dist}\left(g, H^{\infty}(\mathbb{D})[f]\right) \leq \inf _{\delta>0} \sup _{a \in \mathbb{C}} \operatorname{dist}\left(g,\left.\mathbb{C}\right|_{\Omega_{f}(a, \delta)}\right)
$$

where $\operatorname{dist}\left(g,\left.\mathbb{C}\right|_{\Omega_{f}(a, \delta)}\right)$ denotes the distance of $g$ to the constants on $\Omega_{f}(a, \delta)$.

If $\varphi$ is a uniformly continuous function on the plane then $\varphi \circ h$ is close to constant on any set where $h$ is close to constant, in particular on the sets $\Omega_{h}(a, \delta)$. Thus

Corollary 2.4. If $\varphi$ is uniformly continuous on $\mathbb{C}$ then $\varphi \circ h \in H^{\infty}(\mathbb{D})[f]$.

If $\bar{f} \in H^{\infty}(\mathbb{D})$ and $\varphi: \mathbb{C} \rightarrow \mathbb{C}$ is continuous then this implies $\varphi \circ f \in$ $H^{\infty}(\mathbb{D})[f]$. However, this particular consequence is much easier than Theorem 2.1. The function $\varphi$ can be uniformly approximated by a polynomial $P(x, y)$ on the closure of $\bar{f}$ 's range and it's easy to see that if $\bar{f} \in H^{\infty}(\mathbb{D})$ then $\operatorname{Re}(f), \operatorname{Im}(f) \in H^{\infty}(\mathbb{D})[f]$. Thus $P(\operatorname{Re}(f), \operatorname{Im}(f))$ uniformly approximates $\varphi \circ f$ and is in $H^{\infty}(\mathbb{D})[f]$.

Next we consider what happens in Theorem 2.1 when $f$ is real valued.

Corollary 2.5. If $f$ is a real valued, bounded harmonic function on $\mathbb{D}$ and $g \in L^{\infty}(\mathbb{D})$ then

$$
\operatorname{dist}\left(g, H^{\infty}(\mathbb{D})[f]\right)=\inf _{\delta>0} \sup _{a \in \mathbb{R}} \operatorname{dist}\left(g, H^{\infty}\left(\Omega_{f}(a, \delta)\right)\right) .
$$

Proof. The holomorphic function $h$ corresponding to $f$ is $h=f+i f^{*}$. Therefore, if $s, t$ are real, $\Omega_{h}(s+i t, \delta) \subset \Omega_{f}(s, \delta)$, so,

$$
\sup _{a \in \mathbb{C}} \operatorname{dist}\left(g, H^{\infty}\left(\Omega_{h}(a, \delta)\right)\right) \leq \sup _{s \in \mathbb{R}} \operatorname{dist}\left(g, H^{\infty}\left(\Omega_{f}(s, \delta)\right)\right) .
$$

Thus combining Theorem 2.1 and (2.1) gives

$$
\begin{aligned}
\operatorname{dist}\left(g, H^{\infty}(\mathbb{D})[f]\right) & =\inf _{\delta>0} \sup _{a \in \mathbb{C}} \operatorname{dist}\left(g, H^{\infty}\left(\Omega_{h}(a, \delta)\right)\right) \\
& \leq \inf _{\delta>0} \sup _{s \in \mathbb{R}} \operatorname{dist}\left(g, H^{\infty}\left(\Omega_{f}(s, \delta)\right)\right) .
\end{aligned}
$$


To prove the other direction, suppose $\left\{h_{k}\right\} \subset H^{\infty}(\mathbb{D})$ and

$$
F(z)=\sum_{k=0}^{n} h_{k}(z) f^{k}(z) \in H^{\infty}(\mathbb{D})[f]
$$

satisfies

$$
\|g-F\|_{\infty} \leq \operatorname{dist}\left(g, H^{\infty}(\mathbb{D})[f]\right)+\epsilon .
$$

Fix $a$ and let $\tilde{F}(z)=\sum_{k=0}^{n} h_{k}(z) a^{k} \in H^{\infty}(\mathbb{D})$. Then for $z \in \Omega_{f}(a, \delta)$ we have

$$
\begin{aligned}
|g(z)-\tilde{F}(z)| & \leq|g(z)-F(z)|+|F(z)-\tilde{F}(z)| \\
& \leq \operatorname{dist}\left(g, H^{\infty}(\mathbb{D})[f]\right)+\epsilon+\sum_{k=1}^{n}\left|h_{k}(z)\right|\left|f(z)^{k}-a^{k}\right| \\
& \leq \operatorname{dist}\left(g, H^{\infty}(\mathbb{D})[f]\right)+2 \epsilon,
\end{aligned}
$$

if $\delta$ is small enough (depending on $\epsilon,\|f\|_{\infty}$ and $\left\{h_{k}\right\}$, but not on $a$ ). Taking $\epsilon \rightarrow 0$ proves the corollary.

Remark 2.1. Here is a simple example which shows that we must replace $\Omega_{f}$ by $\Omega_{h}$ in Theorem 2.1. Take $f(z)=z$ and $g(z)=\bar{z}$. Then the sets $\Omega_{f}(a, \delta)$ are just small disks $D(a, \delta)$. Since $g$ is uniformly continuous, $g$ is close to holomorphic (in fact close to constant) on every such disk $\Omega_{f}(a, \delta)=D(a, \delta)$, but $g \notin H^{\infty}(\mathbb{D})[f]=H^{\infty}(\mathbb{D})$. Thus Corollary 2.2 is not correct in this case. However, Theorem 2.1 is correct since in this case $h=\bar{f}+i \bar{f}^{*}$ is constant, so the sets $\Omega_{h}(a, \delta)$ can only be empty or the whole disk.

Remark 2.2. For a fixed $a$, the sets $\Omega_{h}(a, \delta)$ decrease as $\delta \rightarrow 0$, so the "inf" is really a limit. Thus we can write

$$
\operatorname{dist}\left(g, H^{\infty}(\mathbb{D})[f]\right)=\lim _{\delta \rightarrow 0} \sup _{a \in \mathbb{C}} \operatorname{dist}\left(g, H^{\infty}\left(\Omega_{h}(a, \delta)\right)\right) .
$$

Similarly, we have an equivalent discrete version

$$
\operatorname{dist}\left(g, H^{\infty}(\mathbb{D})[f]\right)=\lim _{n \rightarrow \infty} \sup _{a \in \mathbb{C}} \operatorname{dist}\left(g, H^{\infty}\left(\Omega_{h}\left(a, \frac{1}{n}\right)\right)\right) .
$$

Of course, similar restatements work for the various corollaries.

Remark 2.3. We claim that in Corollary 2.2 we can interchange the order of the "inf" and the "sup", i.e., for any bounded $f$ we have

$$
\inf _{\delta>0} \sup _{a \in \mathbb{C}} \operatorname{dist}\left(g, H^{\infty}\left(\Omega_{f}(a, \delta)\right)\right)=\sup _{a \in \mathbb{C}} \inf _{\delta>0} \operatorname{dist}\left(g, H^{\infty}\left(\Omega_{f}(a, \delta)\right)\right) .
$$

This follows from the following general fact.

Lemma 2.6. Suppose $Y$ is a compact metric space and $\left\{F_{n}\right\}$ is a sequence of real valued functions on $Y$ such that

(1) $F_{n}(y) \geq F_{n+1}(y)$ for all $n$ and $y$. 
(2) If $\left\{y_{k}\right\} \rightarrow y$ and $n_{k} \rightarrow \infty$ then $\lim \sup _{k \rightarrow \infty} F_{n_{k}}\left(y_{k}\right) \leq F_{n}(y)$ for all $n$. Then

$$
\inf _{n} \sup _{y \in Y} F_{n}(y)=\sup _{y \in Y} \inf _{n} F_{n}(y) .
$$

Proof. The direction $\geq$ is completely general, in fact,

$$
\inf _{x \in X} \sup _{y \in Y} F(x, y) \geq \sup _{y \in Y} \inf _{x \in X} F(x, y),
$$

holds for any real valued function on a product space. To prove it, one simple notes that for a fixed $x_{0}$ and $y_{0}$

$$
F\left(x_{0}, y_{0}\right) \geq \inf _{x \in X} F\left(x, y_{0}\right)
$$

so taking the supremum over $y_{0}$ gives

$$
\sup _{y \in Y} F\left(x_{0}, y\right) \geq \sup _{y \in Y} \inf _{x \in X} F(x, y)
$$

and then taking the infimum over all possible $x_{0}$ 's gives the desired inequality.

To prove the other direction, we need to use our hypotheses. We may choose a sequence $\left\{y_{k}\right\}$ and $\left\{n_{k}\right\}$ so that

$$
F_{n_{k}}\left(y_{k}\right) \rightarrow \sup _{y \in Y} \inf _{n} F_{n}(y)
$$

By compactness of $Y$ we may assume $y_{k} \rightarrow y_{0} \in Y$. Since for a fixed $y$, the sequence $\left\{F_{n}(y)\right\}$ is decreasing, the infimum over $n$ is the same as the limit as $n \rightarrow \infty$. Therefore we may assume (without loss of generality) that $n_{k} \rightarrow \infty$ as $k \rightarrow \infty$. By hypothesis (2) of the lemma, we deduce that for any $n$,

$$
\sup _{y \in Y} \inf _{k} F_{k}(y)=\lim _{k \rightarrow \infty} F_{n_{k}}\left(y_{k}\right) \leq F_{n}\left(y_{0}\right)
$$

for any $n$. Thus

$$
\sup _{y \in Y} \inf _{k} F_{k}(y) \leq \sup _{y \in Y} F_{n}(y)
$$

and so

$$
\sup _{y \in Y} \inf _{k} F_{k}(y) \leq \inf _{n} \sup _{y \in Y} F_{n}(y),
$$

which proves the lemma.

To apply the lemma to our case we simply note that it suffices to assume $\|f\|_{\infty} \leq 1$. Let $Y=\overline{\mathbb{D}}$ and

$$
F_{n}(a)=\operatorname{dist}\left(g, H^{\infty}\left(\Omega_{f}\left(a, \frac{1}{n}\right)\right)\right) .
$$


Then $Y$ is compact and part (1) of the lemma clearly holds (since $\left.\Omega_{f}\left(a, \frac{1}{n+1}\right) \subset \Omega_{f}\left(a, \frac{1}{n}\right)\right)$. To check (2), note that if $a_{k} \rightarrow a$ and $n_{k} \rightarrow \infty$ then $\Omega_{f}\left(a_{k}, \frac{1}{n_{k}}\right) \subset \Omega_{f}\left(a, \frac{1}{n}\right)$ if $\left|a-a_{k}\right|<\frac{1}{2 n}$ and $n_{k}>2 n$ (i.e., if $k$ is large enough). Thus

$$
\begin{aligned}
\inf _{\delta>0} \sup _{a \in \mathbb{C}} \operatorname{dist}\left(g, H^{\infty}\left(\Omega_{f}(a, \delta)\right)\right) & =\inf _{n} \sup _{a \in \mathbb{C}} \operatorname{dist}\left(g, H^{\infty}\left(\Omega_{f}\left(a, \frac{1}{n}\right)\right)\right) \\
& =\sup _{a \in \mathbb{C}} \inf _{n} \operatorname{dist}\left(g, H^{\infty}\left(\Omega_{f}\left(a, \frac{1}{n}\right)\right)\right) \\
& =\sup _{a \in \mathbb{C}} \inf _{\delta>0} \operatorname{dist}\left(g, H^{\infty}\left(\Omega_{f}(a, \delta)\right)\right),
\end{aligned}
$$

as desired.

Remark 2.4. In Theorem 2.1 the "inf" and the "sup" cannot be exchanged in the case of a general $f$. Let $\varphi$ be a conformal mapping from $\mathbb{D}$ to the region $\left\{z=x+i y: 0<y, 0<x<(1+y)^{-1}\right\}$. Let $f=\operatorname{Re}(\varphi)$ so that the corresponding $h$ in Theorem 2.1 equals $\frac{1}{2} \varphi$ and set $u(x, y)=2 x(1+y)$ and $g=u \circ h$. By unwinding the various definitions one can check that

$$
\begin{aligned}
& \sup _{a \in \mathbb{C}} \inf _{\delta>0} \operatorname{dist}\left(g, H^{\infty}\left(\Omega_{h}(a, \delta)\right)\right)=0, \\
& \inf _{\delta>0} \sup _{a \in \mathbb{C}} \operatorname{dist}\left(g, H^{\infty}\left(\Omega_{h}(a, \delta)\right)\right)=1 .
\end{aligned}
$$

Remark 2.5. To see that Corollary 2.3 is really weaker than Corollary 2.2, consider the following example. Let $f=\bar{z}$ and let

$$
U=([0,1] \times[0,1]) \backslash \bigcup_{k=1}^{\infty}\left(\left[0, \frac{1}{2}\right] \times\left\{\frac{1}{n}\right\}\right),
$$

and let $\varphi$ be a conformal mapping of the disk onto $U$ which maps 1 to the prime end containing $\left\{\frac{1}{2}\right\}$. Then $g=\bar{\varphi}$ is close to $\varphi \in H^{\infty}(\mathbb{D})$ in small neighborhoods of $\{1\}$, but $g$ is not close to a constant on any neighborhood of $\{1\}$.

\section{The formula for algebras with several generators.}

In this section we state Theorem 3.1 which gives the formula for $\operatorname{dist}\left(g, H^{\infty}(\mathbb{D})[\mathcal{F}]\right)$ when $\mathcal{F}$ is a countable collection of bounded harmonic functions on $\mathbb{D}$ and $g \in L^{\infty}(\mathbb{D})$.

Given a countable collection $\mathcal{F}=\left\{f_{i}\right\}_{i \in I}$ indexed by the set $I$ (either $I=\mathbb{N}$ or $I=\{1, \ldots, N\}$ for some $N \in \mathbb{N}$ ) and an $\alpha=\left\{a_{i}\right\}_{i \in I} \in \mathbb{C}^{I}$ define

$$
\Omega_{\mathcal{F}}(\alpha, \delta, m)=\bigcap_{f_{k} \in \mathcal{F}, k \leq m} \Omega_{f_{k}}\left(a_{k}, \delta\right) .
$$


Given a collection $\mathcal{F}$ of harmonic functions the corresponding collection of $h$ 's (as described in the previous section) is denoted $\mathcal{H}$. To simplify notation we will sometimes write $\inf _{\delta, m}$ instead of of $\inf _{\delta>0, m \in I}$ and just write $\sup _{\alpha}$ in place of $\sup _{\alpha \in \mathbb{C}^{I}}$.

Theorem 3.1. If $\mathcal{F}$ is countable collection of bounded harmonic functions on $\mathbb{D}$ and $g \in L^{\infty}(\mathbb{D})$ then

$$
\operatorname{dist}\left(g, H^{\infty}(\mathbb{D})[\mathcal{F}]\right)=\inf _{\delta>0, m \in I} \sup _{\alpha \in \mathbb{C}^{I}} \operatorname{dist}\left(g, H^{\infty}\left(\Omega_{\mathcal{H}}(\alpha, \delta, m)\right)\right) .
$$

Remark 3.1. In Remark 2.2 (when $\mathcal{F}=\{f\}$ was a single function) we noted that "inf $f_{\delta}$ " could be replaced by " $\lim _{\delta \rightarrow 0}$ ". Here we could also reinterpret "inf $\delta, m$ " as a limit " $\lim _{(\delta, m) \rightarrow(0,|I|)}$ ", (here $|I|$ denotes the number of elements of the index set $I$ ). The limit could be interpreted in terms of nets (e.g., see page 187 of [12]) indexed by the pairs $(\delta, m) \in(0, \infty) \times I$ with the partial order $(\delta, m) \leq(\eta, n)$ if $\delta \geq \eta$ and $m \leq n$. With this in mind, it is easy to see that

$$
\begin{aligned}
\inf _{\delta>0, m \in I} \sup _{\alpha \in \mathbb{C}^{I}} \operatorname{dist}\left(g, H^{\infty}\left(\Omega_{\mathcal{H}}(\alpha, \delta, m)\right)\right) \\
\quad=\lim _{(\delta, m) \rightarrow(0,|I|)} \sup _{\alpha \in \mathbb{C}^{I}} \operatorname{dist}\left(g, H^{\infty}\left(\Omega_{\mathcal{H}}(\alpha, \delta, m)\right)\right) .
\end{aligned}
$$

One can also view the infimum as a limit in the usual sense by noting that

$$
\begin{aligned}
\inf _{\delta>0, m \in I} \sup _{\alpha \in \mathbb{C}^{I}} \operatorname{dist}\left(g, H^{\infty}\left(\Omega_{\mathcal{H}}(\alpha, \delta, m)\right)\right) \\
\quad=\lim _{n \rightarrow \infty} \sup _{\alpha \in \mathbb{C}^{I}} \operatorname{dist}\left(g, H^{\infty}\left(\Omega_{\mathcal{H}}\left(\alpha, \frac{1}{n}, \min (n,|I|)\right)\right)\right) .
\end{aligned}
$$

Remark 3.2. Corollaries $2.2,2.3$ and 2.5 all have the obvious generalizations to algebras generated by a collection $\mathcal{F}$. For example, if $\mathcal{F}$ consists of bounded anti-holomorphic functions then

$$
\operatorname{dist}\left(g, H^{\infty}(\mathbb{D})[\mathcal{F}]\right)=\inf _{\delta>0, m \in I} \sup _{\alpha \in \mathbb{C}^{I}} \operatorname{dist}\left(g, H^{\infty}\left(\Omega_{\mathcal{F}}(\alpha, \delta, m)\right)\right)
$$

\section{The distance formula for non-harmonic generators.}

We shall see later (Section 6) that if $f$ is bounded and harmonic on $\mathbb{D}$, then $H^{\infty}(\mathbb{D})[f]$ is also generated by a collection of four real-valued functions $\left\{f_{1}, \ldots, f_{4}\right\} \in C(\mathcal{M})$ (see Lemma 6.1). We will also verify a special property 
of these particular functions: given any $\alpha=\left(a_{1}, \ldots, a_{4}\right) \in \mathbb{R}^{4}$, we will show the joint level set

$$
E_{\alpha}=\left\{\zeta \in \mathcal{M}: f_{i}(\zeta)=a_{i}, i=1, \ldots, 4\right\},
$$

is the zero set (in $\mathcal{M}$ ) of some $A \in H^{\infty}(\mathbb{D})$.

Using these facts we will deduce Theorem 3.1 from

Theorem 4.1. Suppose $\mathcal{F}=\left\{f_{i}\right\}_{i \in I} \subset C(\mathcal{M})$ is a countable collection of bounded, real-valued functions with $H^{\infty}$ level sets (i.e., every for $\alpha=\left\{a_{i}\right\} \in$ $\mathbb{R}^{I}$, the set $E_{\alpha}=\left\{\zeta \in \mathcal{M}: f_{i}(\zeta)=a_{i}, i \in I\right\}$ is a countable intersection of zeros sets of $H^{\infty}(\mathbb{D})$ functions). If $g \in L^{\infty}(\mathbb{D})$ then

$$
\operatorname{dist}\left(g, H^{\infty}(\mathbb{D})[\mathcal{F}]\right)=\inf _{\delta>0, m \in I} \sup _{\alpha \in \mathbb{R}} \operatorname{dist}\left(g, H^{\infty}\left(\Omega_{\mathcal{F}}(\alpha, \delta, m)\right)\right) .
$$

Remark 4.1. The argument of Remark 2.3 shows the "inf" and "sup" in Theorem 4.1 may be interchanged, i.e.,

$$
\begin{aligned}
\operatorname{dist}\left(g, H^{\infty}(\mathbb{D})[\mathcal{F}]\right) & =\inf _{\delta>0, m \in I} \sup _{\alpha \in \mathbb{R}^{I}} \operatorname{dist}\left(g, H^{\infty}\left(\Omega_{\mathcal{F}}(\alpha, \delta, m)\right)\right) \\
& =\sup _{\alpha \in \mathbb{R}^{I}} \inf _{\delta>0, m \in I} \operatorname{dist}\left(g, H^{\infty}\left(\Omega_{\mathcal{F}}(\alpha, \delta, m)\right)\right) .
\end{aligned}
$$

To repeat the proof in this setting, we assume (without loss of generality) that all the functions in $\mathcal{F}$ are bounded by 1 . Take $Y=\overline{\mathbb{D}}^{I}$, which is easily seen to be compact with the metric

$$
d(\alpha, \beta)=d\left(\left\{a_{i}\right\},\left\{b_{i}\right\}\right)=\inf \left\{\frac{1}{n}:\left|a_{i}-b_{i}\right| \leq \frac{1}{n}, i=1, \ldots, \min (n,|I|)\right\} .
$$

Then set

$$
F_{n}(y)=\operatorname{dist}\left(g, H^{\infty}\left(\Omega_{\mathcal{F}}\left(y, \frac{1}{n}, \min (n,|I|)\right)\right)\right) .
$$

One verifies (1) and (2) of Lemma 2.6 just as before using the facts that

$$
\Omega_{\mathcal{F}}\left(y, \frac{1}{n+1}, \min (n+1,|I|)\right) \subset \Omega_{\mathcal{F}}\left(y, \frac{1}{n}, \min (n,|I|)\right),
$$

and

$$
\Omega_{\mathcal{F}}\left(y_{k}, \frac{1}{n_{k}}, \min \left(n_{k},|I|\right)\right) \subset \Omega_{\mathcal{F}}\left(y, \frac{1}{n}, \min (n,|I|)\right),
$$

if $d\left(y, y_{k}\right)<\frac{1}{2 n}$ and $n_{k}>2 n$.

This argument also shows that the "inf" and "sup" may be exchanged in Theorem 3.1 if the collection $\mathcal{F}$ consists of anti-holomorphic functions 
(or, more generally, if the associated collection $\mathcal{H}$ of holomorphic functions consists of bounded functions).

Remark 4.2. We mentioned above that if $f$ is bounded and harmonic then $H^{\infty}(\mathbb{D})[f]$ is also generated by $H^{\infty}(\mathbb{D})$ and a collection of real valued functions. I do not know whether $H^{\infty}(\mathbb{D})[f]$ can always be generated by $H^{\infty}(\mathbb{D})$ and some collection of real valued harmonic functions, but this seems unlikely.

\section{The distance formula for $g \in C(\mathcal{M})$.}

So far we have been careful to state the distance formula in such a way that it makes sense for any $g \in L^{\infty}(\mathbb{D})$. In this section we will specialize Theorem 4.1 to the case when $g$ has a continuous extension to $\mathcal{M}$ (which we will still call $g$ ). In this case Theorem 4.1 has a more compact statement which is very similar to Shilov's theorem (described in the introduction).

We start by reviewing a few facts about $\mathcal{M}$, the maximal ideal space of $H^{\infty}(\mathbb{D})$. A basis for the topology is generated by sets of the form

$$
\tilde{\Omega}_{f}(a, \delta)=\{\zeta \in \mathcal{M}:|f(\zeta)-a|<\delta\}
$$

where $f \in H^{\infty}(\mathbb{D}), a \in \mathbb{C}$ and $\delta>0$. For a collection of functions $\mathcal{F} \subset C(\mathcal{M})$ we define

$$
\tilde{\Omega}_{\mathcal{F}}(\alpha, \delta, m)=\bigcap_{f_{k} \in \mathcal{F}, k \leq m} \tilde{\Omega}_{f_{k}}\left(a_{k}, \delta\right),
$$

just as before, but now it is a subset of $\mathcal{M}$ instead of $\mathbb{D}$. It is clear that

$$
\tilde{\Omega}_{\mathcal{F}}(\alpha, \delta, m) \cap \mathbb{D}=\Omega_{\mathcal{F}}(\alpha, \delta, m) .
$$

(From now on, $\tilde{\Omega}$ will always denote a set in $\mathcal{M}$ and $\Omega$ the corresponding intersection with $\mathbb{D}$.) Conversely, the fact that $\mathbb{D}$ is dense in $\mathcal{M}$ (Carleson's corona theorem) implies that

$$
\tilde{\Omega}_{\mathcal{F}}(\alpha, \delta, m)=\bigcup_{\eta<\delta} \overline{\Omega_{\mathcal{F}}(a, \eta, m)}
$$

(the closure is in $\mathcal{M}$ ). Note that the zero set of a function on $\mathcal{M}$ need not be the closure of its zero set on $\mathbb{D}$, but it is always equal to

$$
\{\zeta \in \mathcal{M}: f(\zeta)=0\}=\bigcap_{\delta} \overline{\Omega_{f}(0, \delta)}=\bigcap_{\delta} \tilde{\Omega}_{f}(0, \delta),
$$

(the closure is taken in $\mathcal{M})$. Furthermore, a function $f \in H^{\infty}\left(\Omega_{\mathcal{F}}(\alpha, \delta)\right.$ ) has a unique continuous extension to $\tilde{\Omega}_{\mathcal{F}}(\alpha, \delta)$. 
Given a compact subset $E \subset \mathcal{M}$ let $H(E)$ be the functions $f$ on $E$ which have a continuous extension $F$ to open neighborhood $U$ of $E$ such that $F$ is bounded and holomorphic on $U \cap \mathbb{D}$. Let $H_{\text {loc }}^{\infty}(E)$ denote the closure of $H(E)$ in $C(E)$ with respect to the sup norm on $E$. Clearly $\left.H^{\infty}(\mathbb{D})\right|_{E} \subset H(E) \subset$ $H_{\mathrm{loc}}^{\infty}(E)$.

Next we want to rewrite the distances in Theorem 3.1 and Theorem 4.1 in terms of distances to $H_{\text {loc }}^{\infty}(E)$. Suppose that $E \subset \mathcal{M}$ can be written in the form

$$
E=\bigcap_{i \in I}\left\{\zeta \in \mathcal{M}: f_{i}(\zeta)=0\right\}=\bigcap_{\delta>0, m \in I} \tilde{\Omega}_{\mathcal{F}}(a, \delta, m)
$$

for some collection functions $\mathcal{F}=\left\{f_{i}\right\}_{i \in I} \subset C(\mathcal{M})$ (i.e., $E$ is a joint level set in $\mathcal{M})$. Then it is easy to see that

$$
\operatorname{dist}\left(g, H_{\mathrm{loc}}^{\infty}(E)\right)=\inf _{\delta>0, m \in I} \operatorname{dist}\left(g, H^{\infty}\left(\Omega_{\mathcal{F}}(0, \delta, m)\right)\right) .
$$

Therefore Theorem 4.1 implies

Theorem 5.1. Suppose $\mathcal{F}=\left\{f_{i}\right\}_{i \in I}$ is as in Theorem 4.1. If $g \in C(\mathcal{M})$ then

$$
\operatorname{dist}\left(g, H^{\infty}(\mathbb{D})[\mathcal{F}]\right)=\sup _{\alpha \in \mathbb{R}^{I}} \operatorname{dist}\left(\left.g\right|_{E_{\alpha}}, H_{\text {loc }}^{\infty}\left(E_{\alpha}\right)\right),
$$

where $E_{\alpha}=\left\{\zeta \in \mathcal{M}: f_{i}(\zeta)=a_{i}, i \in I\right\}$.

Remark 5.1. We will prove later (Lemma 7.1) that if $E \subset \mathcal{M}$ is a finite intersection of zero sets of functions in $H^{\infty}(\mathbb{D})$ then $\left.H^{\infty}(\mathbb{D})\right|_{E}=H(E)$, and therefore we have $\overline{\left.H^{\infty}(\mathbb{D})\right|_{E}}=H_{\text {loc }}^{\infty}(E)$. It would be interesting to know for which sets $E \subset \mathcal{M}$ we have $\overline{\left.H^{\infty}(\mathbb{D})\right|_{E}}=H_{\mathrm{loc}}^{\infty}(E)$. If $K \subset \mathbb{D}$ is compact this is Runge's theorem: every function holomorphic on a neighborhood of $K$ can be approximated by a polynomial iff $K$ has connected complement. This condition is sometimes stated by saying $K$ is polynomially convex, i.e., $K=\hat{K}$ where

$$
\hat{K} \equiv\left\{z:|p(z)| \leq \sup _{K}|p| \text { for all polynomials } p\right\} .
$$

J. Garnett has suggested that the appropriate condition for the $H^{\infty}$ problem should be the analogous convexity condition: $E=\hat{E}$ where

$$
\hat{E} \equiv\left\{\zeta \in \mathcal{M}:|f(\zeta)| \leq \sup _{E}|f| \text { for all } f \in H^{\infty}\right\} .
$$




\section{Reducing Theorem 3.1 to Theorem 4.1.}

Now that we have introduced all the notation and stated the results we are ready to start proving the theorems. In this section we show how to deduce Theorem 3.1 from Theorem 4.1. Theorem 4.1 will be proven in the following two sections. We start with,

Lemma 6.1. If $f$ is a bounded harmonic function on $\mathbb{D}$, let $h=\frac{1}{2}\left(\bar{f}+i \bar{f}^{*}\right)$. Then $H^{\infty}(\mathbb{D})[f]$ is generated by four real valued functions $\mathcal{C}=\left\{f_{1}, \ldots, f_{4}\right\}$ which satisfy the following conditions:

(1) $f_{i} \in C(\mathcal{M}), \quad i=1,2,3,4$.

(2) If $\alpha=\left\{a_{1}, \ldots, a_{4}\right\} \in \mathbb{R}^{4}$, then there is an $A \in H^{\infty}(\mathbb{D})$ such that

$$
\cap_{i=1}^{4}\left\{\zeta \in \mathcal{M}: f_{i}(\zeta)=a_{i}\right\}=\{\zeta \in \mathcal{M}: A(\zeta)=0\} .
$$

In other words, the common level sets are zero sets for $H^{\infty}(\mathbb{D})$. A more precise version of this is the following; there is an absolute $C<\infty$ and an $A \in H^{\infty}(\mathbb{D})$ such that

$$
C^{-1} \leq \frac{|A(z)|}{\sum_{j=1}^{4}\left|a_{i}-f_{i}(z)\right|} \leq C .
$$

(3) We have the following equality

$$
\inf _{\delta} \sup _{\alpha \in \mathbb{C}} \operatorname{dist}\left(g, H^{\infty}\left(\Omega_{h}(a, \delta)\right)\right)=\inf _{\delta} \sup _{\alpha \in \mathbb{R}^{4}} \operatorname{dist}\left(g, H^{\infty}\left(\Omega_{\mathcal{C}}(\alpha, \delta, 4)\right)\right) .
$$

In the rest of the paper, the notation $A \sim B$ means that $A / B$ is bounded and bounded away from zero. Thus the inequality in part (2) of the lemma could be written

$$
|A(z)| \sim \sum_{j=1}^{4}\left|a_{i}-f_{i}(z)\right| .
$$

To deduce Theorem 3.1, note that parts (1) and (2) of the lemma imply that Theorem 4.1 can be applied to $H^{\infty}(\mathbb{D})[f]=H^{\infty}(\mathbb{D})[\mathcal{C}]$, giving

$$
\begin{aligned}
\operatorname{dist}\left(g, H^{\infty}(\mathbb{D})[f]\right) & =\operatorname{dist}\left(g, H^{\infty}(\mathbb{D})[\mathcal{C}]\right) \\
& =\inf _{\delta} \sup _{\alpha \in \mathbb{R}^{4}} \operatorname{dist}\left(g, H^{\infty}\left(\Omega_{\mathcal{C}}(\alpha, \delta, 4)\right)\right) .
\end{aligned}
$$

Now apply part (3) of the lemma to get

$$
\operatorname{dist}\left(g, H^{\infty}(\mathbb{D})[f]\right)=\inf _{\delta} \sup _{\alpha \in \mathbb{C}} \operatorname{dist}\left(g, H^{\infty}\left(\Omega_{h}(a, \delta)\right)\right),
$$

which is Theorem 3.1 in the case of one generator. An extra argument is required to deduce the general case of Theorem 3.1 (the case of several 
generating functions) and we will give this at the end of this section. In the remainder of this section we will prove the lemma.

Let $f=u+i v=\operatorname{Re}(f)+i \operatorname{Im}(f)$ and $h=\frac{1}{2}\left(\bar{f}+i \bar{f}^{*}\right)$. We start by using the observation of Axler and Shields [1] that if $f$ is harmonic on $\mathbb{D}$ and $\|f\|_{\infty} \leq \pi / 4$ then the algebra $H^{\infty}(\mathbb{D})[f]$ is also generated by the functions

$$
G(z)=e^{i \operatorname{Re}(h(z))}=e^{i\left(u(z)+v^{*}(z)\right)}=e^{i f(z)} e^{v(z)+i v^{*}(z)},
$$

and

$$
F(z)=e^{i \operatorname{Im}(h(z))}=e^{i\left(v(z)-u^{*}(z)\right)}=e^{f(z)} e^{-\left(u(z)+i u^{*}(z)\right)} .
$$

Lemma 6.2. If $f$ is bounded and harmonic on $\mathbb{D}$ and $G, F$ are as above, then $G, F \in H^{\infty}(\mathbb{D})[f]$ and $H^{\infty}(\mathbb{D})[f]=H^{\infty}(\mathbb{D})[G]=H^{\infty}(\mathbb{D})[F]$.

Proof. First observe that $e^{i f(z)} \in H^{\infty}(\mathbb{D})[f]$ because it can be approximated by finite sums of the series

$$
1+i f(z)+\frac{1}{2}(i f(z))^{2}+\cdots+\frac{1}{n !}(i f(z))^{n}+\ldots,
$$

which converges uniformly to $e^{i f(z)}$ since $f$ is bounded. Moreover, the function $e^{v(z)+i v^{*}(z)}$ is bounded and holomorphic on $\mathbb{D}$ (since $v$ is bounded). Thus by (6.1) $G$ is the product of functions in $H^{\infty}(\mathbb{D})[f]$, so is in $H^{\infty}(\mathbb{D})[f]$. Similarly for $F$.

To see why $G$ generates $H^{\infty}(\mathbb{D})[f]$, we use our assumption that $\|f\|_{\infty} \leq$ $\pi / 4$. Then $e^{i f(z)}$ can only take values on the arc $\left\{e^{i \theta}:|\theta|<\frac{\pi}{4}\right\}$. This arc is a compact subset of the disk $D(1,1)$, so the power series for $\log z$ centered at 1 converges uniformly on this arc. Thus $f(z)=-i \log \left(e^{i f(z)}\right)$ is in $H^{\infty}(\mathbb{D})[G]$, as desired. The same proof works for $F$.

Since the complex conjugate of $G$ is given by

$$
\bar{G}(z)=G(z)^{-1}=e^{-i \operatorname{Re}(h(z))}=e^{-i\left(u(z)+v^{*}(z)\right)}=e^{-i f(z)} e^{-\left(v(z)+i v^{*}(z)\right)},
$$

the same argument as above shows $\bar{G} \in H^{\infty}(\mathbb{D})[f]$. Thus

$$
\operatorname{Re}(G)=\frac{1}{2}(G+\bar{G}), \quad \operatorname{Im}(G)=\frac{1}{2 i}(G-\bar{G}),
$$

are real valued functions in the algebra $H^{\infty}(\mathbb{D})[f]$ and these two functions (with $H^{\infty}(\mathbb{D})$ ) generate the algebra.

We now start the proof of Lemma 6.1. We begin with part (1). Define

$$
\begin{aligned}
\mathcal{C} & =\left\{f_{1}, \ldots, f_{2}\right\} \\
& =\{\operatorname{Re}(G), \operatorname{Im}(G), \operatorname{Re}(F), \operatorname{Im}(F)\} \\
& =\{\cos (\operatorname{Re}(h)), \sin (\operatorname{Re}(h)), \cos (\operatorname{Im}(h)), \sin (\operatorname{Im}(h))\} .
\end{aligned}
$$


Then $\mathcal{C} \subset H^{\infty}(\mathbb{D})[f] \subset C(\mathcal{M})$, so part (1) of Lemma 6.1 is satisfied and $H^{\infty}(\mathbb{D})[f]$ is generated by $\mathcal{C}$ (by Lemma 6.2 ).

Next we verify part (2) of Lemma 6.1, i.e., the level sets are holomorphic zero sets. Note that the sets where the quadruple is constant are the same as

$$
\{z \in \mathbb{D}: h(z) \in a+2 \pi \mathbb{Z}+i 2 \pi \mathbb{Z}\},
$$

i.e., they are a countable union of level sets of $h$. More precisely, we claim that

Lemma 6.3. Given $a \in \mathbb{C}$ let

$$
\left\{a_{1}, a_{2}, a_{3}, a_{4}\right\}=\{\cos (\operatorname{Re}(a)), \sin (\operatorname{Re}(a)), \cos (\operatorname{Im}(a)), \sin (\operatorname{Im}(a))\},
$$

and given $h$ on the disk let $\left\{f_{1}, f_{2}, f_{3}, f_{4}\right\}$ be as in (6.3). Let $\mathcal{L}$ be the lattice $2 \pi(\mathbb{Z}+i \mathbb{Z})$. Then

$$
\operatorname{dist}(h(z), \mathcal{L}+a) \sim \sum_{j=1}^{4}\left|a_{i}-f_{i}(z)\right| .
$$

Proof. First note that for any $w, a \in \mathbb{C}$, we have

$$
|w-a| \sim|\operatorname{Re}(w)-\operatorname{Re}(a)|+|\operatorname{Im}(w)-\operatorname{Im}(a)|,
$$

(i.e., the $L^{1}$ and $L^{2}$ norms on $\mathbb{R}^{2}$ are equivalent) and

$$
\begin{aligned}
\operatorname{dist}(w, a+\mathcal{L}) \sim \operatorname{dist}(\operatorname{Re}(w) & , \operatorname{Re}(a)+2 \pi \mathbb{Z}) \\
+ & \operatorname{dist}(\operatorname{Im}(w), \operatorname{Im}(a)+2 \pi \mathbb{Z}) .
\end{aligned}
$$

Similarly, for $x, y \in \mathbb{R}$,

$$
\begin{aligned}
\operatorname{dist}(x, y+2 \pi \mathbb{Z}) & \sim\left|e^{i x}-e^{i y}\right| \\
& \sim|\cos (x)-\cos (y)|+|\sin (x)-\sin (y)| .
\end{aligned}
$$

Combining (6.3), (6.4) and (6.5) proves the lemma.

That the quadruple level set is also the level set of a single bounded holomorphic function is immediate from the following (see Lemma 3.1 of [2]).

Lemma 6.4. Suppose $h \in B M O A(\mathbb{D})$. Let $\mathcal{L}=2 \pi(\mathbb{Z}+i \mathbb{Z})$ be the lattice of Gaussian integers (times $2 \pi$ ). Then there exists a bounded holomorphic function $A$ on the unit disk and a constant $C$ (depending only on the BMO norm of $h$ ) such that for all $z \in \mathbb{D}$,

$$
C^{-1} \operatorname{dist}(h(z), \mathcal{L}) \leq|A(z)| \leq C \operatorname{dist}(h(z), \mathcal{L}) .
$$


Combining Lemma 6.4 and Lemma 6.3 proves part (2) of Lemma 6.1. Next we verify part (3) of Lemma 6.1 Fix an $a \in \mathbb{C}$ and let

$$
\begin{aligned}
\alpha & =\left\{a_{1}, a_{2}, a_{3}, a_{4}\right\} \\
& =\left\{\operatorname{Re}\left(e^{i \operatorname{Re}(a)}\right), \operatorname{Im}\left(e^{i \operatorname{Re}(a)}\right), \operatorname{Re}\left(e^{i \operatorname{Im}(a)}\right), \operatorname{Im}\left(e^{i \operatorname{Im}(a)}\right)\right\} \\
& =\{\cos (\operatorname{Re}(a)), \sin (\operatorname{Re}(a)), \cos (\operatorname{Im}(a)), \sin (\operatorname{Im}(a))\}
\end{aligned}
$$

Let $X \subset \mathbb{R}^{4}$ be the set of all $\alpha$ 's which have this form. Note that $X$ is a compact set which contains the range of the map

$$
\left(f_{1}, f_{2}, f_{3}, f_{4}\right): \mathcal{M} \rightarrow \mathbb{C}
$$

Lemma 6.3 says there is a $C<\infty$ such that

$$
\Omega_{\mathcal{C}}(\alpha, \delta / C, 4) \subset \bigcup_{w \in \mathbb{Z}+i \mathbb{Z}} \Omega_{h}(a+2 \pi w, \delta) \subset \Omega_{\mathcal{C}}(\alpha, C \delta, 4) .
$$

Note that if $\left\{\Omega_{j}\right\}$ are disjoint open sets then

$$
\operatorname{dist}\left(g, H^{\infty}\left(\cup_{j} \Omega_{j}\right)\right)=\sup _{j} \operatorname{dist}\left(g, H^{\infty}\left(\Omega_{j}\right)\right) .
$$

Since the union in (6.6) is disjoint if $\delta<\pi$, we get (for all small $\delta$ ),

$$
\begin{aligned}
\operatorname{dist}\left(g, H^{\infty}\left(\Omega_{\mathcal{C}}(\alpha, \delta / C, 4)\right)\right) & \leq \sup _{w \in \mathbb{Z}+i \mathbb{Z}} \operatorname{dist}\left(g, H^{\infty}\left(\Omega_{h}((a+2 \pi w, \delta))\right)\right) \\
& \leq \operatorname{dist}\left(g, H^{\infty}\left(\Omega_{\mathcal{C}}(\alpha, C \delta, 4)\right)\right)
\end{aligned}
$$

Therefore,

$$
\begin{aligned}
\sup _{\alpha \in X} \operatorname{dist}\left(g, H^{\infty}\left(\Omega_{\mathcal{C}}(\alpha, \delta / C, 4)\right)\right) & \leq \sup _{a \in \mathbb{C}} \sup _{w \in \mathbb{C}} \operatorname{dist}\left(g, H^{\infty}\left(\Omega_{h}((a+2 \pi w, \delta))\right)\right) \\
& \leq \sup _{\alpha \in X} \operatorname{dist}\left(g, H^{\infty}\left(\Omega_{\mathcal{C}}(\alpha, C \delta, 4)\right)\right) .
\end{aligned}
$$

Thus taking the infimum over $\delta$ gives equality.

Also observe that,

$$
\sup _{a \in \mathbb{C}} \sup _{w \in \mathbb{C}} \operatorname{dist}\left(g, H^{\infty}\left(\Omega_{h}(((a+2 \pi w), \delta))\right)\right)=\sup _{a \in \mathbb{C}} \operatorname{dist}\left(g, H^{\infty}\left(\Omega_{h}((a, \delta))\right)\right),
$$

hence,

(6.7) $\inf _{\delta>0} \sup _{\alpha \in X} \operatorname{dist}\left(g, H^{\infty}\left(\Omega_{\mathcal{C}}(\alpha, \delta, 4)\right)\right)=\inf _{\delta>0} \sup _{a \in \mathbb{C}} \operatorname{dist}\left(g, H^{\infty}\left(\Omega_{h}(a, \delta)\right)\right)$. 
Finally, we wish to replace $X$ by $\mathbb{R}^{4}$. If $\alpha \notin X$ then $\alpha$ is not in the range of the quadruple, so either $\Omega_{\mathcal{C}}(\alpha, \delta, 4)$ is empty or $\Omega_{\mathcal{C}}(\alpha, \delta, 4) \subset \Omega_{\mathcal{C}}(\beta, 2 \delta, 4)$ for some $\beta \in X$. Thus either $\operatorname{dist}\left(g, H^{\infty}\left(\Omega_{\mathcal{C}}(\alpha, \delta, 4)\right)\right)=0$ or

$$
\operatorname{dist}\left(g, H^{\infty}\left(\Omega_{\mathcal{C}}(\alpha, \delta, 4)\right)\right) \leq \operatorname{dist}\left(g, H^{\infty}\left(\Omega_{\mathcal{C}}(\beta, 2 \delta, 4)\right)\right)
$$

for some $\beta \in X$. Therefore,

$$
\inf _{\delta>0} \sup _{\alpha \in \mathbb{R}^{4}} \operatorname{dist}\left(g, H^{\infty}\left(\Omega_{\mathcal{C}}(\alpha, \delta, 4)\right)\right)=\inf _{\delta>0} \sup _{\alpha \in X} \operatorname{dist}\left(g, H^{\infty}\left(\Omega_{\mathcal{C}}(\alpha, \delta, 4)\right)\right) .
$$

Now (6.7) and (6.8) prove part (3) of Lemma 6.1.

We have now proven Theorem 3.1 (given Theorem 4.1) when the algebra is generated by one function. To do the general case, suppose $\mathcal{F}=\left\{f_{i}\right\}_{i \in I}$ is a countable family of bounded harmonic functions and let

$$
\mathcal{H}=\left\{h_{i}\right\}_{i \in I}=\left\{\bar{f}_{i}+i \bar{f}_{i}^{*}\right\},
$$

be the family of corresponding holomorphic functions. We form a countable collection $\mathcal{C}$ by converting each $f_{i} \in \mathcal{F}$ into a quadruple $\left\{f_{i, j}\right\}_{j=1,2,3,4}$ as above. Then $\mathcal{C}$ generates $H^{\infty}(\mathbb{D})[\mathcal{F}]$ and property $(1)$ holds as before. To prove (2) we simply note that

$$
\left\{\zeta \in \mathcal{M}: f_{i, j}(\zeta)=a_{i, j}, i \in I, j=1,2,3,4\right\}=\bigcap_{i \in I}\left\{\zeta \in \mathcal{M}: A_{i}(\zeta)=0\right\}
$$

where $A_{i} \in H^{\infty}(\mathbb{D})$ is the function associated to $f_{i}$ by Lemma 6.1. Thus common level sets of the collection $\mathcal{C}$ are countable intersections of $H^{\infty}$ zero sets, as required in Theorem 4.1. Finally, we have to verify (3). To do this we simply note that Lemma 6.3 implies

$$
\Omega_{\mathcal{C}}(\alpha, \delta / C, 4 m) \subset \bigcup_{w \in \mathbb{Z}+i \mathbb{Z}} \Omega_{\mathcal{H}}(a+2 \pi w, \delta, m) \subset \Omega_{\mathcal{C}}(\alpha, C \delta, 4 m),
$$

(index $\left\{f_{i, j}\right\}$ in the obvious way). This gives (as before),

$$
\inf _{\delta, m} \sup _{\alpha \in \mathbb{C}^{\infty}} \operatorname{dist}\left(g, H^{\infty}\left(\Omega_{\mathcal{H}}(\alpha, \delta, m)\right)\right)=\inf _{\delta, m} \sup _{\alpha \in \mathbb{R}^{\infty}} \operatorname{dist}\left(g, H^{\infty}\left(\Omega_{\mathcal{C}}(\alpha, \delta, m)\right)\right),
$$

as desired. Thus Theorem 4.1 implies Theorem 3.1 in the general case.

\section{Proof of Theorem 4.1.}

In this section we reduce the proof of Theorem 4.1 to a lemma involving approximation in $H^{\infty}(\mathbb{D})$. We prove the lemma in the following section. 
Without loss of generality we may assume that all the functions in $\mathcal{F}$ are bounded by 1 . We start with the easy direction,

$$
\operatorname{dist}\left(g, H^{\infty}(\mathbb{D})[\mathcal{F}]\right) \geq \inf _{\delta, m} \sup _{\alpha} \operatorname{dist}\left(g, H^{\infty}\left(\Omega_{\mathcal{F}}(\alpha, \delta, m)\right)\right) .
$$

Fix some $\epsilon>0$. By definition we can choose a finite collection of functions $\left\{h_{j, k}\right\} \in H^{\infty}(\mathbb{D})$ so that $G=\sum h_{j, k} f_{j}^{k} \in H^{\infty}(\mathbb{D})[\mathcal{F}]$ satisfies

$$
\|g(z)-G(z)\|_{\infty} \leq \operatorname{dist}\left(g, H^{\infty}(\mathbb{D})[\mathcal{F}]\right)+\epsilon .
$$

Let $S=\sum_{j, k}\left\|h_{j, k}\right\|_{\infty}$, let $M$ be the largest value of $j$ used in the definition of $G$ and let $N$ be the largest power of any $f_{j}$ in the sum defining $G$. Fix $\alpha=\left\{a_{i}\right\} \in \mathbb{R}^{I}$. Then on $\Omega_{F}(\alpha, \delta, M)$ we have

$$
\begin{aligned}
\left\|g(z)-\sum_{k} h_{j, k}(z) a_{j}^{k}\right\|_{\infty} \\
\leq\left\|g(z)-\sum h_{j, k}(z) f_{j}^{k}(z)\right\|_{\infty}+\left\|\sum_{k} h_{j, k}(z)\left(f_{j}^{k}(z)-a_{j}^{k}\right)\right\|_{\infty} \\
\leq \operatorname{dist}\left(g, H^{\infty}(\mathbb{D})[\mathcal{F}]\right)+\epsilon+\left\|\sum_{k} h_{j, k}(z)\left(f_{j}^{k}(z)-a_{j}^{k}\right)\right\|_{\infty} \\
\leq \operatorname{dist}\left(g, H^{\infty}(\mathbb{D})[\mathcal{F}]\right)+\epsilon+2 S N \sup _{j \leq M}\left\|f_{j}\right\|_{\infty} \delta \\
\leq \operatorname{dist}\left(g, H^{\infty}(\mathbb{D})[\mathcal{F}]\right)+2 \epsilon,
\end{aligned}
$$

if $\delta$ is small enough. Since $\sum h_{j, k}(z) a_{j}^{k}$ is holomorphic on the whole disk we get,

$$
\begin{aligned}
\inf _{\delta, m} \operatorname{dist}\left(g, H^{\infty}\left(\Omega_{\mathcal{F}}(\alpha, \delta, m)\right)\right) & \leq\|g(z)-G(z)\|_{\infty} \\
& \leq \operatorname{dist}\left(g, H^{\infty}(\mathbb{D})[\mathcal{F}]\right)+2 \epsilon .
\end{aligned}
$$

Since this holds for any $\alpha \in \mathbb{R}^{I}$ we get

$$
\sup _{\alpha} \inf _{\delta, m} \operatorname{dist}\left(g, H^{\infty}\left(\Omega_{\mathcal{F}}(\alpha, \delta, m)\right)\right) \leq \operatorname{dist}\left(g, H^{\infty}(\mathbb{D})[\mathcal{F}]\right) .
$$

Now use Remark 4.1 to exchange the "sup" and the "inf" and we obtain (7.1).

Next we must prove the opposite inequality

$$
\operatorname{dist}\left(g, H^{\infty}(\mathbb{D})[\mathcal{F}]\right) \leq \inf _{\delta, m} \sup _{\alpha} \operatorname{dist}\left(g, H^{\infty}\left(\Omega_{\mathcal{F}}(\alpha, \delta, m)\right)\right) .
$$

The main idea in the proof is given by the following lemma, which is implicit in Carleson's solution of the corona problem (see [6, Chapter VIII]). 
Lemma 7.1. Suppose $\mathcal{A}=\left\{A_{k}\right\} \subset H^{\infty}(\mathbb{D})$ and $E=\cap_{k}\left\{\zeta \in \mathcal{M}: A_{k}(\zeta)=\right.$ $0\}$. Then $\left.H^{\infty}(\mathbb{D})\right|_{E}=H(E)$. More precisely, given a bounded holomorphic function $G$ on $\Omega=\Omega_{\mathcal{A}}(0, \delta, m)$ there exists $\epsilon>0$ and $\tilde{G} \in H^{\infty}(\mathbb{D})$ such that $\|\tilde{G}\|_{\infty} \leq C=C(\mathcal{A}, \delta, m)$ and such that $|G(z)-\tilde{G}(z)| \leq C \sum_{k=1}^{m}\left|A_{k}(z)\right|$ for $z \in \Omega_{\mathcal{A}}(0, \epsilon, m)$.

We will prove the lemma in the next section. In the remainder of this section we will give the proof of inequality (7.2) using Lemma 7.1. The proof is a standard argument involving compactness and partitions of unity.

Fix $\epsilon>0, \alpha=\left\{a_{i}\right\}_{i \in I} \in[-1,1]^{I}$ and a $g \in L^{\infty}(\mathbb{D})$. Let $E_{\alpha}=\cap_{i \in I}\{\zeta \in$ $\left.\mathcal{M}: f_{i}(\zeta)=a_{i}\right\}$ denote the level set in $\mathcal{M}$ corresponding to $\alpha$. We will construct an $H \in H^{\infty}(\mathbb{D})[\mathcal{F}]$ so that

$$
\|g-H\|_{\infty} \leq \inf _{\delta, m} \sup _{\alpha} \operatorname{dist}\left(g, H^{\infty}\left(\Omega_{\mathcal{F}}(\alpha, \delta, m)\right)\right)+2 \epsilon .
$$

Clearly, if we can construct such a $H$, we will have proven (7.2).

For each $\alpha \in \mathbb{R}^{I}$, choose $\delta, m$ and $G_{\alpha} \in H^{\infty}\left(\Omega_{\mathcal{F}}(\alpha, \delta, m)\right)$ so that

$$
\left\|\left.g(z)\right|_{\Omega_{\mathcal{F}}(\alpha, \delta, m)}-G_{\alpha}(z)\right\|_{\infty}<\inf _{\delta, m} \sup _{\alpha} \operatorname{dist}\left(g, H^{\infty}\left(\Omega_{\mathcal{F}}(\alpha, \delta, m)\right)\right)+\epsilon .
$$

Let $\mathcal{A}=\left\{A_{1}, \ldots\right\}$ be the sequence of bounded holomorphic functions associated to the level set $E_{\alpha}$ by assumption. The hypotheses of Theorem 4.1 say that the collections

$$
\left\{\tilde{\Omega}_{\mathcal{A}}(0, \delta, m)\right\}_{\delta>0, m \in I}, \quad\left\{\tilde{\Omega}_{\mathcal{F}}(\alpha, \delta, m)\right\}_{\delta>0, m \in I},
$$

both form a basis of neighborhoods for the set $E_{\alpha}$. In other words, given any open neighborhood $U$ of $E_{\alpha}$ there are $\delta>0$ and $m \in I$ such that

$$
\begin{aligned}
& \tilde{\Omega}_{\mathcal{A}}(0, \delta, m) \subset U, \\
& \tilde{\Omega}_{\mathcal{F}}(\alpha, \delta, m) \subset U .
\end{aligned}
$$

In particular given $\delta$ and $m$ there are $\delta^{\prime}, \delta^{\prime \prime}$ and $m^{\prime}, m^{\prime \prime}$ such that

$$
E_{\alpha} \subset \Omega_{\mathcal{F}}\left(\alpha, \delta^{\prime \prime}, m^{\prime \prime}\right) \subset \tilde{\Omega}_{\mathcal{A}}\left(0, \delta^{\prime}, m^{\prime}\right) \subset \tilde{\Omega}_{\mathcal{F}}(\alpha, \delta, m) .
$$

This is just simple point set topology using the fact that $\mathcal{M}$ is a compact Hausdorff space.

Now use Lemma 7.1 to obtain a $\tilde{G}_{\alpha} \in H^{\infty}(\mathbb{D})$ such that

$$
\left|G_{\alpha}(z)-\tilde{G}_{\alpha}(z)\right| \leq C \sum_{k=1}^{m}\left|A_{k}(z)\right|, \quad z \in \Omega_{\mathcal{A}}\left(0, \epsilon, m^{\prime}\right),
$$


where $C$ depends on $h, \alpha, \delta^{\prime}, m^{\prime}$ but not on $z$. Thus

$$
\begin{aligned}
\left|g(z)-\tilde{G}_{\alpha}(z)\right| & \leq\left|g(z)-G_{\alpha}(z)\right|+\left|G_{\alpha}(z)-\tilde{G}_{\alpha}(z)\right| \\
& \leq\left|g(z)-G_{\alpha}(z)\right|+C \sum_{k=1}^{m}|A(z)| \\
& \leq\left|g(z)-G_{\alpha}(z)\right|+C m \eta
\end{aligned}
$$

for $\zeta \in \tilde{\Omega}_{A}\left(0, \eta, m^{\prime}\right)$. Thus if $\eta<\epsilon / C m$ we get

$$
\begin{aligned}
\left|g(z)-\tilde{G}_{\alpha}(z)\right| & \leq\left|g(z)-G_{\alpha}(z)\right|+\epsilon \\
& \leq \operatorname{dist}\left(g, H^{\infty}\left(\Omega_{\mathcal{F}}(\alpha, \delta, m)\right)\right)+2 \epsilon .
\end{aligned}
$$

By (7.3) we can choose $\delta^{\prime \prime}$ and $m^{\prime \prime}$ so that

$$
\tilde{\Omega}_{\mathcal{F}}\left(\alpha, \delta^{\prime \prime}, m^{\prime \prime}\right) \subset \tilde{\Omega}_{\mathcal{A}}\left(0, \eta, m^{\prime}\right) .
$$

Every point of $\mathcal{M}$ is in some level set $E_{\alpha}$ and so the sets $\left\{\tilde{\Omega}_{\mathcal{F}}\left(\alpha, \delta^{\prime \prime}, m^{\prime \prime}\right)\right\}_{\alpha \in \mathbb{D}^{\prime}}$ form an open cover of $\mathcal{M}$. Since $\mathcal{M}$ is compact, we can find a finite subcover $\left\{\tilde{\Omega}_{j}\right\}_{j=1}^{N}=\left\{\tilde{\Omega}_{\mathcal{F}}\left(\alpha_{j}, \delta_{j}, m_{j}\right)\right\}_{j=1}^{N}$. Let $\Omega_{j}=\mathbb{D} \cap \tilde{\Omega}_{j}$ for $j=1, \ldots, N$. For each $j$ we construct (as above) a $\tilde{G}_{j} \in H^{\infty}(\mathbb{D})$ so that

$$
\left|g(z)-\tilde{G}_{j}(z)\right| \leq \inf _{\delta, m} \sup _{\alpha} \operatorname{dist}\left(g, H^{\infty}\left(\Omega_{\mathcal{F}}(\alpha, \delta, m)\right)\right)+2 \epsilon, \quad z \in \Omega_{j} .
$$

Let $M$ denote the largest $m_{j}$ used in this cover. For each $j=1, \ldots, N$, suppose $\alpha_{j}=\left\{a_{i, j}\right\} \in \mathbb{R}^{I}$, and define

$$
B_{j}=\left\{\left(x_{1}, \ldots, x_{m_{j}}\right) \in \mathbb{R}^{m_{j}}:\left|x_{i}-a_{i, j}\right|<\delta, i=1, \ldots m_{j}\right\},
$$

and define $U_{j}=B_{j} \times \mathbb{R}^{M-m_{j}} \subset \mathbb{R}^{M}$. Consider the continuous map from $\mathcal{M}$ to $\mathbb{R}^{M}$ given by the $M$-tuple $\left(f_{1}, \ldots, f_{M}\right)$. Since $\mathcal{M}$ is compact, the image is compact. By definition, the sets $\left\{U_{j}\right\}$ form a finite open cover of this compact set, so we may choose a continuous partition of unity $\left\{\psi_{j}\right\}$ of this set which is subordinate to the cover $\left\{U_{j}\right\}$.

$H^{\infty}(\mathbb{D})[\mathcal{F}]$ contains every function of the form $\psi\left(f_{1}, \ldots, f_{M}\right)$, with $\psi$ continuous on $\mathbb{R}^{M}$. To prove this, we recall that on any compact subset of $\mathbb{R}^{M}$ the polynomials are dense in the continuous functions. Thus $\psi$ can be uniformly approximated by a polynomial $P\left(x_{1}, \ldots, x_{M}\right)$. Then $P\left(f_{1}, \ldots, f_{M}\right)$, uniformly approximates $\psi\left(f_{1}, \ldots, f_{M}\right)$, so $\psi\left(f_{1}, \ldots, f_{M}\right)$ is in the algebra generated by the $f_{i}$ 's (in particular, it is in $H^{\infty}(\mathbb{D})[\mathcal{F}]$ ). This is the only place that we use our assumption that the functions $\left\{f_{1}, \ldots, f_{M}\right\}$ are real valued.

Thus $\left\{\tilde{\psi}_{j}\right\}_{j=1}^{N}=\left\{\psi_{j}\left(f_{1}, \ldots, f_{M}\right)\right\}_{j=1}^{N} \subset H^{\infty}(\mathbb{D})[\mathcal{F}]$ is a partition of unity on $\mathcal{M}$, subordinate to the cover $\left\{\tilde{\Omega}_{j}\right\}_{j=1}^{N}$. In particular, these functions form 
a partition of unity when restricted to the disk. For a fixed $z, \sum_{j=1}^{N} \tilde{\psi}_{j}(z)=1$, so

$$
\sum_{j=1}^{N} \tilde{G}_{j}(z) \tilde{\psi}_{j}(z)
$$

is a convex combination of the complex numbers $\left\{\tilde{G}_{j}(z)\right\}_{j=1}^{N}$. By Equation (7.4) these points all lie in a disk of radius

$$
\inf _{\delta, m} \sup _{\alpha} \operatorname{dist}\left(g, H^{\infty}\left(\Omega_{\mathcal{F}}(\alpha, \delta, m)\right)\right)+2 \epsilon
$$

around $g(z)$. Hence the convex combination also lies in this disk, i.e.,

$$
\left\|g(z)-\sum_{j=1}^{N} \tilde{G}_{j}(z) \tilde{\psi}_{j}(z)\right\|_{\infty} \leq \inf _{\delta, m} \sup _{\alpha} \operatorname{dist}\left(g, H^{\infty}\left(\Omega_{\mathcal{F}}(\alpha, \delta, m)\right)\right)+2 \epsilon,
$$

as desired. Thus $H=\sum_{j=1}^{N} \tilde{G}_{j} \tilde{\psi}_{j} \in H^{\infty}(\mathbb{D})[\mathcal{F}]$ is the desired approximation. This completes the proof of Theorem 4.1, except for the proof of Lemma 7.1.

\section{Proof of Lemma 7.1.}

First we prove Lemma 7.1 when there is only one function, $\mathcal{A}=\{A\}$. We will then obtain the general case by induction.

By assumption the function $G$ in the lemma is defined on some neighborhood of $E$ of the form $\tilde{\Omega}_{A}(0, \delta)$. Fix this value of $\delta$. By Carleson's corona construction (e.g., [6], Theorem VIII.5.1) given $A \in H^{\infty}(\mathbb{D})$ with $\|A\|_{\infty} \leq 1$ and a $\delta>0$ there is a $\psi \in C^{\infty}(\mathbb{D})$ such that

(1) $0 \leq \psi \leq 1$

(2) $\psi(z)=1$ if $|A(z)|>\delta$

(3) $\psi(z)=0$ if $|A(z)|<\epsilon=\epsilon(\delta)$,

(4) $|\nabla \psi(z)| \leq C(1-|z|)^{-1}$,

(5) $\operatorname{supp}(\nabla \psi)$ is contained within a unit hyperbolic neighborhood of a Carleson contour $\Gamma \subset \mathbb{D}$. (Recall that $\Gamma$ is a Carleson contour if there is a $C<\infty$ such that the arclength of $\Gamma \cap D(x, r) \leq C r$ for any $x \in \partial \mathbb{D}$.)

Define $H(z)=(1-\psi(z)) G(z)$ on $U \cap \mathbb{D}$ and let $H=0$ elsewhere on $\mathbb{D}$. Then $H$ is smooth on the whole disk and is holomorphic except in the region

$$
\{z \in \mathbb{D}: \bar{\partial} \psi(z) \neq 0\} \subset\{z \in \mathbb{D}: \epsilon(\delta)<|A(z)|<\delta\} .
$$

Moreover, $H=G$ on the set $\{z \in \mathbb{D}:|A(z)|<\epsilon(\delta)\}$. 
Suppose we could find a function $\varphi$ on the unit disk, bounded by $C$ and so that $F=H+A \varphi$ is holomorphic. Then $F$ satisfies

$$
\|F\|_{\infty} \leq\|H\|_{\infty}+\|\varphi\|_{\infty}\|A\|_{\infty}<\infty
$$

so $F \in H^{\infty}(\mathbb{D})$. Furthermore,

$$
|F(z)-H(z)| \leq\|\varphi\|_{\infty}|A(z)| \leq C|A(z)| .
$$

On the set $\{z \in \mathbb{D}:|A(z)| \leq \epsilon\}$ we have $H=(1-\psi) G=G$, so

$$
|F(z)-G(z)| \leq|F(z)-H(z)|+|H(z)-G(z)| \leq C|A(z)| .
$$

Therefore, $F$ satisfies the conditions of the lemma (in the case $m=1$ ). So to prove the $m=1$ case of the lemma it suffices to find a bounded $\varphi$ so that $H+A \varphi$ is holomorphic.

We do this by solving a $\bar{\partial}$ equation. For a smooth function $f$ on the disk define $\bar{\partial} f=\frac{1}{2}\left(f_{x}+i f_{y}\right)$. Recall that $f$ is holomorphic on the disk iff $\bar{\partial} f \equiv 0$ (this is just the Cauchy-Riemann equations in a slightly different form). In other words, we want to find a bounded solution $\varphi$ of the equation

$$
\bar{\partial}(H+\varphi A) \equiv 0 .
$$

Since $\bar{\partial}$ is linear and $A$ is holomorphic we can rewrite this as

$$
\bar{\partial} H+A \bar{\partial} \varphi \equiv 0
$$

or equivalently,

$$
\bar{\partial} \varphi=-\frac{\bar{\partial} H}{A}=\frac{-\bar{\partial}((1-\psi) G)}{A}=-\frac{G}{A} \bar{\partial} \psi
$$

By (5) above, the right hand side is supported in a unit neighborhood of a Carleson contour $\Gamma$. Furthermore, $G$ is bounded above (say by $M$ ) on the disk and $A$ is bounded below by $\epsilon$ on this neighborhood. Thus $-\frac{G}{A} \bar{\partial} \psi$ is supported in unit neighborhood of a Carleson contour and satisfies

$$
\left|\frac{G(z) \bar{\partial} \psi(z)}{A(z)}\right| \leq C M \epsilon^{-1}(1-|z|)^{-1} .
$$

Thus a bounded solution of (8.1) exists by the following result.

Lemma 8.1. Suppose $b$ is a smooth function on $\mathbb{D}$ such that

(1) b supported in a unit hyperbolic neighborhood of a Carleson contour $\Gamma$,

(2) $b(z) \leq \frac{M}{1-|z|}$. 
Then there exists a bounded, smooth $F$ such that $\bar{\partial} F=b$ and $\|F\|_{\infty} \leq$ $C(\Gamma, M)$.

This is Lemma 3.3 of Garnett and Jones' paper [7] (actually they prove a more general version, replacing the disk with more general domains). A proof is also given on page 789 of [2].

This completes the proof of Lemma 7.1 for the case of a single $A$.

Next we deduce the general case using induction. First note that if $A$ is a bounded, analytic function and $\delta>0$ then each component of $\Omega=$ $\Omega_{A}(0, \delta) \subset \mathbb{D}$, is simply connected. Otherwise there would be a point $x$ on its boundary, surrounded by a loop $\gamma$ in $\Omega$. Applying the maximum principle to $A(z)$ on the subdomain of $\mathbb{D}$ bounded by $\gamma$ and containing $x$ we deduce $|A(x)|<\delta$, a contradiction.

Fix some $m$ and for $0 \leq k \leq m-1$ define

$$
U_{k}=\Omega_{\mathcal{A}}(0, \delta, m-k)=\bigcap_{j=1}^{m-k} \Omega_{A_{j}}(0, \delta) .
$$

Thus

$$
\Omega_{\mathcal{A}}(0, \delta, m)=U_{0} \subset U_{2} \subset \cdots \subset U_{m-1} \subset \mathbb{D} \text {. }
$$

Since each component of a finite intersection of simply connected domains is also simply connected, we deduce that each $U_{k}$ is a union of simply connected components.

Suppose we are given $G \in H^{\infty}\left(\Omega_{\mathcal{A}}(0, \delta, m)\right)=H^{\infty}\left(U_{0}\right)$. Since each component $\Omega$ of $U_{1}$ is simply connected it is conformally equivalent to the unit disk so by the previous case of Lemma 7.1 there is an $\epsilon_{0}>0$ and a bounded holomorphic function $\tilde{G}$ in $H^{\infty}(\Omega)$ so that

$$
|G(z)-\tilde{G}(z)| \leq C A_{m}(z)
$$

for

$$
z \in \Omega \cap\left\{z \in \mathbb{D}:\left|A_{m}(z)\right| \leq \epsilon_{0}\right\}=U_{0}^{\prime} \subset U_{0} .
$$

Applying this procedure separately to each component of $U_{1}$ gives us a function $G_{1}$ defined on all of $U_{1}$ with the same estimate.

By induction we see that for each $k=1, \ldots, m$ there is an $\epsilon_{k}>0$ and a $G_{k} \in H^{\infty}\left(U_{k}\right)$ so that

$$
\left|G_{k-1}(z)-G_{k}(z)\right| \leq C\left|A_{k}(z)\right|
$$

for

$$
z \in U_{k-1}^{\prime}=\left\{z \in U_{k}:\left|A_{k}(z)\right| \leq \epsilon_{k}\right\}
$$


Continuing for $m$ steps we get a bounded holomorphic function $G_{m}$ on the unit disk so that

$$
\left|G-G_{m}\right| \leq \sum_{k=1}^{m}\left|G_{k}-G_{k+1}\right| \leq C \sum_{k=1}^{m}\left|A_{k}\right|
$$

for

$$
z \in \Omega_{\mathcal{F}}(0, \epsilon, m) \subset \cap_{k=0}^{m-1} U_{k}^{\prime},
$$

where $\epsilon=\min _{k} \epsilon_{k}$. This proves Lemma 7.1.

\section{An application: the Bourgain closure of $H^{\infty}(\mathbb{D})[\mathcal{F}]$.}

The following application was suggested by conversations with Joe Cima and Dechao Zheng.

Given Banach algebras $A \subset B$, the Bourgain closure of $A$ in $B$ is defined as

$$
A_{b}=\left\{g \in B:\left\{f_{n}\right\} \subset A, f_{n} \rightarrow 0 \text { weakly, implies } \operatorname{dist}\left(g f_{n}, A\right) \rightarrow 0\right\} .
$$

This notion was introduced by Cima and Timoney in [5]. Some recent papers on the topic include $[3,4,8,9,10,11,14,15]$. In [8] Ghatage, Sun and Zheng prove that the Bourgain closure of $H^{\infty}(\mathbb{D})$ in $C(\mathcal{M})$ is $H^{\infty}(\mathbb{D})[\bar{z}]=H^{\infty}(\mathbb{D})+C(\overline{\mathbb{D}})$. In $[6]$, Cima, Stroethoff and Yale show that its Bourgain closure in $L^{\infty}(\mathbb{D})$ is $H^{\infty}(\mathbb{D})[\bar{z}]+V$ where $V=\left\{g \in L^{\infty}(\mathbb{D})\right.$ : $\left.\lim _{r \rightarrow 1}\|g\|_{L^{\infty}(\mathbb{D} \backslash r \mathbb{D})}=0\right\}$. Here we will use Theorem 4.1 to compute the Bourgain closure of $H^{\infty}(\mathbb{D})[\mathcal{F}]$. First we verify two simple facts.

Lemma 9.1. Suppose $\mathcal{F}$ satisfies the conditions of Theorem 4.1. If $\left\{f_{n}\right\}$ is a sequence in $L^{\infty}(\mathbb{D})$ and $\operatorname{dist}\left(f_{n}, H^{\infty}(\mathbb{D})[\mathcal{F}]\right) \rightarrow 0$ then for every $\epsilon>0$ there exist $n_{0}, m_{0}, \delta_{0}$ such that $n \geq n_{0}, m \geq m_{0}, \delta \leq \delta_{0}$ implies

$$
\sup _{\alpha} \operatorname{dist}\left(f_{n}, H^{\infty}\left(\Omega_{\mathcal{F}}(\alpha, \delta, m)\right)\right)<\epsilon .
$$

Proof. As usual, we may assume $\|f\|_{\infty} \leq 1$ for every $f \in \mathcal{F}$. Suppose the lemma fails. Then there are $\delta_{k} \rightarrow 0, n_{k}, m_{k} \rightarrow \infty$ and $\alpha_{k} \in \mathbb{D}^{I}$ so that

$$
\operatorname{dist}\left(f_{n_{k}}, H^{\infty}\left(\Omega_{\mathcal{F}}\left(\alpha_{k}, \delta_{k}, m_{k}\right)\right)\right) \geq \epsilon .
$$

We may use the compactness of $\mathbb{D}^{I}$ (see Remark 4.1) to pass to a subsequence such that $\alpha_{k} \rightarrow \alpha \in \mathbb{D}^{I}$, and using the fact that $\Omega_{\mathcal{F}}\left(\alpha_{k}, \delta_{k}, m_{k}\right) \subset$ $\Omega_{\mathcal{F}}(\alpha, \delta, m)$ if $k$ is large enough, we see that $\operatorname{dist}\left(f_{n_{k}}, H^{\infty}\left(\Omega_{\mathcal{F}}(\alpha, \delta, m)\right)\right) \geq \epsilon$ for all $\delta$ and $m$ if $k$ is sufficiently large. Therefore

$$
\inf _{\delta, m} \sup _{\alpha} \operatorname{dist}\left(f_{n_{k}}, H^{\infty}\left(\Omega_{\mathcal{F}}(\alpha, \delta, m)\right)\right) \geq \epsilon
$$


so $\operatorname{dist}\left(f_{n_{k}}, H^{\infty}(\mathbb{D})[\mathcal{F}]\right) \nrightarrow \nrightarrow 0$ by Theorem 4.1 , a contradiction.

Lemma 9.2. Suppose $\mathcal{F}=\left\{f_{1}, \ldots, f_{m}\right\}$ is a finite collection of real valued functions on the unit disk such that the common level sets

$$
E_{\alpha}=\left\{z \in \mathbb{D}: f_{i}(z)=a_{i}, i=1, \ldots, m\right\}
$$

(where $\alpha=\left(a_{1}, \ldots a_{m}\right) \in \mathbb{R}^{m}$ ) are countable. Then

$$
\operatorname{dist}\left(g, H^{\infty}\left(\Omega_{\mathcal{F}}(\alpha, \delta, m)\right)\right)=\lim _{\eta \rightarrow 0} \operatorname{dist}\left(g, H^{\infty}\left(\Omega_{\mathcal{F}}(\alpha, \delta, m) \backslash \overline{\Omega_{\mathcal{F}}(\alpha, \eta, m)}\right)\right) .
$$

Proof. First we claim that

$$
\begin{aligned}
A & \equiv \lim _{\eta \rightarrow 0} \operatorname{dist}\left(g, H^{\infty}\left(\Omega_{\mathcal{F}}(\alpha, \delta, m) \backslash \overline{\Omega_{\mathcal{F}}(\alpha, \eta, m)}\right)\right) \\
& =\operatorname{dist}\left(g, H^{\infty}\left(\Omega_{\mathcal{F}}(\alpha, \delta) \backslash E_{\alpha}\right)\right) \equiv B .
\end{aligned}
$$

The " $A \leq B$ " direction is clear since

$$
\Omega_{\mathcal{F}}(\alpha, \delta, m) \backslash \overline{\Omega_{\mathcal{F}}(\alpha, \eta, m)} \subset \Omega_{\mathcal{F}}(\alpha, \delta, m) \backslash E_{\alpha} .
$$

To prove the other direction take a sequence

$$
g_{n} \in H^{\infty}\left(\Omega_{\mathcal{F}}(\alpha, \delta, m) \backslash \Omega_{\mathcal{F}}(\alpha, \delta / n, m)\right)
$$

with $\left\|g-g_{n}\right\| \rightarrow A$. Since the $g_{n}$ are defined on a increasing sequence of sets, normal families implies they converge uniformly on compact sets to $g_{\infty} \in H^{\infty}\left(\Omega_{\mathcal{F}}(\alpha, \delta, m) \backslash E_{\alpha}\right)$ and that $\left\|g-g_{\infty}\right\| \leq A$. Thus we get $B \leq A$.

But by hypothesis, $E_{\alpha}$ is a countable subset of $\Omega_{\mathcal{F}}(\alpha, \delta, m)$ and hence removable for bounded analytic functions. Thus

$$
\operatorname{dist}\left(g, H^{\infty}\left(\Omega_{\mathcal{F}}(\alpha, \delta, m)\right)\right)=\operatorname{dist}\left(g, H^{\infty}\left(\Omega_{\mathcal{F}}(\alpha, \delta, m) \backslash E_{\alpha}\right)\right),
$$

and we are done.

Theorem 9.3. If $\mathcal{U}$ is a countable collection of bounded harmonic functions on the unit disk (at least one of which is not holomorphic), then $\left(H^{\infty}(\mathbb{D})[\mathcal{U}]\right)_{b}=H^{\infty}(\mathbb{D})[\mathcal{U}]\left(\right.$ in both $L^{\infty}(\mathbb{D})$ and $\left.C(\mathcal{M})\right)$.

Proof: We may as well assume $\mathcal{U}$ contains no holomorphic functions. In Lemma 6.1 we proved that the algebra generated by $H^{\infty}(\mathbb{D})$ and a countable collection of bounded harmonic, non-holomorphic functions can also be 
generated by $H^{\infty}(\mathbb{D})$ and a countable collection of non-constant real valued functions. Let $\mathcal{F}=\left\{f_{i}\right\}_{i \in I}$ be the collection of real valued functions corresponding to $\mathcal{U}$. Thus it is enough to show $H^{\infty}(\mathbb{D})[\mathcal{F}]=\left(H^{\infty}(\mathbb{D})[\mathcal{F}]\right)_{b}$.

By a result in [5], $A \subset A_{b}$ in general, so $H^{\infty}(\mathbb{D})[\mathcal{F}] \subset\left(H^{\infty}(\mathbb{D})[\mathcal{F}]\right)_{b}$. To prove the opposite inclusion suppose $g \notin H^{\infty}(\mathbb{D})[\mathcal{F}]$. We will construct a sequence $\left\{F_{n}\right\} \subset H^{\infty}(\mathbb{D})[\mathcal{F}]$ converging weakly to zero so that $\operatorname{dist}\left(g F_{n}, H^{\infty}(\mathbb{D})[\mathcal{F}]\right) \nrightarrow 0$. By Theorem 4.1 there exists an $\alpha=\left\{a_{i}\right\} \in \mathbb{R}^{I}$ and an $\epsilon_{0}>0$ such that

$$
\operatorname{dist}\left(g, H^{\infty}\left(\Omega_{\mathcal{H}}(\alpha, \delta, m)\right)\right) \geq \epsilon_{0},
$$

for every $\delta>0$ and $m \in I$. For each $n \in \mathbb{N}$ let $m_{n}=\min (n,|I|)$. By Lemma 9.2, given any $\delta_{n}>0$ there must be an $\eta_{n}>0$ so that

$$
\operatorname{dist}\left(g, H^{\infty}\left(\Omega_{\mathcal{F}}\left(\alpha, \delta_{n}, m_{n}\right) \backslash \overline{\Omega_{\mathcal{F}}\left(\alpha, \eta_{n}, m_{n}\right)}\right)\right) \geq \frac{\epsilon_{0}}{2}
$$

We may also assume that $\eta_{n}<\delta_{n}$. To apply Lemma 9.2 we need to know the common level sets of $\left\{f_{1}, \ldots, f_{m_{n}}\right\}$ (in the disk) are at most countable. Using the definition of $\left\{f_{n}\right\}$ from the $\left\{u_{n}\right\}$ given in Section 6 , it is clear that the common level sets of $f_{1}, \ldots, f_{4}$ (the functions which correspond to $u_{1}$ ) is a countable set; in fact it is a countable union of level sets for a certain holomorphic function in the disk which is non-constant if $u_{1}$ is not holomorphic. Thus taking $m \geq 4$ implies Lemma 9.2 may be applied.

Now choose a sequence $\left\{\delta_{n}\right\}$ by induction tending to zero and so that the corresponding sequence $\left\{\eta_{n}\right\}$ satisfies $4 \delta_{n+1} \leq \eta_{n}$. Let $\psi_{n}$ be a continuous function on $R^{m_{n}}$ so that $\psi_{n}(x)=1$ on $\left\{x=\left(x_{1}, \ldots, x_{m_{n}}\right): \eta_{n} \leq\left|x_{i}-a_{i}\right| \leq\right.$ $\left.\delta_{n}\right\}$ and $\psi_{n}(z)=0$ on $\left\{x:\left|x_{i}-a_{i}\right| \geq 2 \delta_{n}\right\}$ and $\left\{z:\left|x_{i}-a_{i}\right| \leq \eta_{n} / 2\right\}$. Then by Theorem $4.1, F_{n}=\psi_{n}\left(f_{1}, \ldots, f_{m_{n}}\right) \in H^{\infty}(\mathbb{D})[\mathcal{F}]$. (We don't really need Theorem 4.1: see the argument in the paragraphs following (7.4).) Observe that

$$
F_{n}(z)=1, z \in \Omega_{\mathcal{F}}\left(\alpha, \delta_{n}, m_{n}\right) \backslash \overline{\Omega_{\mathcal{F}}\left(\alpha, \eta_{n}, m_{n}\right)}
$$

From equations (6.1) and (6.2) it is clear that the functions in $\mathcal{F}$ are all non-constant (if the functions in $\mathcal{U}$ are not holomorphic). Thus the range of any function in $\mathcal{F}$ is connected and not a single point. So if we are considering a point $a \in \mathbb{R}^{I}$ with $\left(a_{1}, \ldots a_{m_{n}}\right)$ in the closure of the range of $\left(f_{1}, \ldots, f_{m_{n}}\right)$ in $R^{m_{n}}$, the functions $F_{n}$ will be non-constant for all large enough $n$ (since the support of $\psi_{n}$ hits the range but does not contain the range).

Since the $F_{n}$ 's are uniformly bounded and have disjoint supports, they 
tend weakly to zero. Recall that $\delta_{n}<\frac{1}{n}$ and use (9.1) to deduce

$$
\begin{aligned}
\operatorname{dist}\left(g F_{n}, \Omega_{\mathcal{F}}\left(\alpha, \frac{1}{n}, m_{n}\right)\right) & \geq \operatorname{dist}\left(g F_{n}, H^{\infty}\left(\Omega_{\mathcal{F}}\left(\alpha, \delta_{n}, m_{n}\right)\right) \backslash \overline{\Omega_{\mathcal{F}}\left(\alpha, \eta_{n}, m_{n}\right)}\right) \\
& \geq \operatorname{dist}\left(g, H^{\infty}\left(\Omega_{\mathcal{F}}\left(\alpha, \delta_{n}, m_{n}\right)\right) \backslash \overline{\Omega_{\mathcal{F}}\left(\alpha, \eta_{n}, m_{n}\right)}\right) \\
& \geq \frac{\epsilon_{0}}{2} .
\end{aligned}
$$

Thus

$$
\lim _{n \rightarrow \infty} \sup _{\alpha} \operatorname{dist}\left(g F_{n}, \Omega_{\mathcal{F}}\left(\alpha, \frac{1}{n}, m_{n}\right)\right) \geq \epsilon_{0} / 2 .
$$

By Remark 3.1 this shows

$$
\inf _{\delta, m} \sup _{\alpha} \operatorname{dist}\left(g F_{n}, \Omega_{\mathcal{F}}(\alpha, \delta, m)\right) \geq \frac{\epsilon_{0}}{2}
$$

and so by Theorem 4.1,

$$
\operatorname{dist}\left(g F_{n}, H^{\infty}(\mathbb{D})[\mathcal{F}]\right) \geq \frac{\epsilon_{0}}{2},
$$

for all $n$. Therefore $g \notin\left(H^{\infty}(\mathbb{D})[\mathcal{F}]\right)_{b}$ and the Theorem 9.3 is proven.

One interesting feature of Theorem 9.3 is that the Bourgain closure of $H^{\infty}(\mathbb{D})$ is strictly larger than that of $H^{\infty}(\mathbb{D})[\bar{z}]$ (as subalgebras of $L^{\infty}(\mathbb{D}$ ); they are equal as subalgebras of $C(\mathcal{M})$ ). Comparing the calculation of $H^{\infty}(\mathbb{D})_{b}$ in [4] to the proof of Theorem 9.3 shows that this disparity is due to the fact that weak convergence to 0 in $H^{\infty}(\mathbb{D})$ implies uniform convergence on compacta. Thus if $\left\{f_{n}\right\} \in H^{\infty}(\mathbb{D})$ converges weakly to $0,\left\{g f_{n}\right\}$ converges uniformly on compacta to 0 and we get no restrictions on $g$ on compacta. However, there are sequences $f_{n} \in H^{\infty}(\mathbb{D})[\bar{z}]$ which converge weakly to 0 but not uniformly on compacta and these impose restrictions on $g$ at all points of the disk.

\section{Another application: the Axler-Shields theorem.}

Theorem 2.1 was originally motivated by trying to understand the following result of Axler and Shields [1]:

Theorem 10.1. If $f$ is bounded and harmonic on $\mathbb{D}$, but not holomorphic, then $H^{\infty}(\mathbb{D})[f]$ contains $C(\overline{\mathbb{D}})$, the uniformly continuous functions on $\mathbb{D}$.

Proof. Here we give a short proof using Corollary 2.3. Suppose $g \in C(\overline{\mathbb{D}})$. Let $h=f+i \bar{f}^{*}$. Then $h$ is holomorphic but not constant (because $f$ is not holomorphic). We claim that the Euclidean diameter of the components of 
$\Omega_{h}(a, \delta)=\{z \in \mathbb{D}:|h(z)-a|<\delta\}$ tend to zero with $\delta$. If not, then there are connected sets $\left\{F_{n}\right\}$ in $\mathbb{D}$ with $\operatorname{diam}\left(F_{n}\right) \geq \epsilon_{0}>0$ and constants $a_{n}$ such that $\left|h(z)-a_{n}\right| \leq e^{-n}$ for $z \in F_{n}$. The harmonic measure of the $F_{n}$ 's from any point in $D(0,1 / 2)$ is clearly bounded away from zero, so by the subharmonicity of $\log |h(z)-a|$ we deduce $\log \left|h(z)-a_{n}\right| \leq C \log e^{-n}=-C n$ for all $z \in D(0,1 / 2)$. We deduce $h$ is constant on the disk, contrary to assumption, and so have proved the claim that all components are small. Since $g$ is uniformly continuous on the disk, $g$ is close to a constant on each component. Thus $g \in H^{\infty}(\mathbb{D})[\bar{f}]$ by Corollary 2.3.

Let $A(\mathbb{D})=H^{\infty}(\mathbb{D}) \cap C(\overline{\mathbb{D}})$ denote the disk algebra. As a consequence of the result above, Axler and Shields deduced the following corollary: if $f \in C(\overline{\mathbb{D}})$ is harmonic but not holomorphic on $\mathbb{D}$ then $A(\mathbb{D})[f]=C(\overline{\mathbb{D}})$. However, there seems to be no direct proof of the corollary (without using Theorem 10.1) and they asked for an explanation of this. One possible reason is that the behavior of the algebra depends on the behavior of the level sets of $h=\bar{f}+i \bar{f}^{*}$, not those of $f$. To prove the distance formula for continuous $f$ reduces to showing $H(E)=\left.H^{\infty}(\mathbb{D})\right|_{E}$ for level sets of functions in $Q A=H^{\infty} \cap V M O$ instead of $H^{\infty}$, but this does not seem significantly easier. Thus although the introduction of $h$ makes our formula look more complicated, it explains this observation of Axler and Shields.

\section{References}

[1] S. Axler and A. Shields, Algebras generated by analytic and harmonic functions, Indiana Univ. Math. J., 39 (1987), 631-638.

[2] C.J. Bishop, Approximating continuous functions by holomorphic and harmonic functions, Trans. Amer. Math. Soc., 311 (1989), 781-811.

[3] J. Cima, S. Janson, and K. Yale, Completely continuous Hankel operators on $H^{\infty}$ and Bourgain algebras, Proc. Amer. Math. Soc., 105 (1989), 121-125.

[4] J. Cima, K. Stroethoff, and K. Yale, Bourgain algebras on the disk, Pacific J. Math., 160 (1993), 27-41.

[5] J. Cima and R. Timoney, The Dunford-Pettis property for certain planar uniform algebras, Michigan Math. J., 34 (1987), 99-104.

[6] J.B. Garnett, Bounded analytic functions, Academic Press, 1981.

[7] J.B. Garnett and P.W. Jones, The Corona theorem for Denjoy domains, Acta Math., 155 (1985), 27-40.

[8] P. Ghatage, S. Sun, and D. Zheng, A remark on Bourgain algebras on the disk, Proc. Amer. Math. Soc., 114 (1992), 395-398.

[9] P. Gorkin, K. Izuchi, and R. Mortini, Bourgain algebras of Douglas algebras, Can. J. Math., 44 (1992), 797-804.

[10] C. Guillory and K. Izuchi, Minimal envelopes of Douglas algebras and Bourgain algebras, Houston J. Math., 19 (1993), 201-222. 
[11] K. Izuchi, Bourgain algebras of the disk, polydisk and ball algebras, Duke Math. J., 66 (1992), 503-519.

[12] J.R. Munkres, Topology; a first course, Prentice-Hall, 1975.

[13] C. Rickart, General theory of Banach algebras, Van Nostrand, 1960.

[14] K. Yale, Bourgain algebras, In K. Jarosz, editor, Proceedings of conference on function spaces, Lecture Notes in Pure and Appl. Math. vol. 136. Marcel Dekker, 1992.

[15] D. Zheng, Toeplitz and Hankel operators on the Bergman spaces of bounded symmetric domains and the Bargman-Fock-Segal spaces, and some disk algebras, $\mathrm{PhD}$ thesis, SUNY, Stony Brook, 1992.

Received November 23, 1993 and revised June 14, 1995.

SUNY STONY BROOK

STONY BRook, NY 11794

E-mail address: bishop@math.sunysb.edu 




\section{PACIFIC JOURNAL OF MATHEMATICS}

Volume $174 \quad$ No. $1 \quad$ May 1996

A distance formula for algebras on the disk

CHRISTOPHER J. BISHOP

Rigidity of isotropic maps

FERNANDO CUKIERMAN

The Schwartz space of a general semisimple Lie group. V. Schwartz

class wave packets

REBECCA A. HERB

Rational polynomials with a $\mathbf{C}^{*}$-fiber

SHULIM KALIMAN

Linear combinations of logarithmic derivatives of entire functions with 195 applications to differential equations

Joseph B. Miles and JoHn Rossi

Factorization problems in the invertible group of a homogeneous $C^{*}$-algebra

N. Christopher PHiLlips

Higher order estimates in complex interpolation theory

RICHARD ROCHBERG

Braid commutators and Vassiliev invariants

TED STANFORD

On the Cauchy problem for a singular parabolic equation 